\title{
Synchronization and Electronic Circuit Application of Hidden Hyperchaos in a Four-Dimensional Self-Exciting Homopolar Disc Dynamo without Equilibria
}

\author{
Yu Feng, ${ }^{1}$ Zhouchao Wei, ${ }^{1,2,3}$ Uğur Erkin Kocamaz, ${ }^{4}$ Akif Akgül, ${ }^{5}$ and Irene Moroz ${ }^{3}$ \\ ${ }^{1}$ Guangxi Colleges and Universities Key Laboratory of Complex System Optimization and Big Data Processing, \\ Yulin Normal University, Yulin 537000, China \\ ${ }^{2}$ School of Mathematics and Physics, China University of Geosciences, Wuhan 430074, China \\ ${ }^{3}$ Mathematical Institute, University of Oxford, Oxford OX2 6GG, UK \\ ${ }^{4}$ Department of Computer Technologies, Vocational School of Karacabey, Uludă̆ University, Karacabey, 16700 Bursa, Turkey \\ ${ }^{5}$ Department of Electrical and Electronic Engineering, Faculty of Technology, Sakarya University, Adapazarl, Turkey
}

Correspondence should be addressed to Zhouchao Wei; weizhouchao@163.com

Received 3 February 2017; Accepted 19 March 2017; Published 31 May 2017

Academic Editor: Karthikeyan Rajagopal

Copyright ( 2017 Yu Feng et al. This is an open access article distributed under the Creative Commons Attribution License, which permits unrestricted use, distribution, and reproduction in any medium, provided the original work is properly cited.

We introduce and investigate a four-dimensional hidden hyperchaotic system without equilibria, which is obtained by augmenting the three-dimensional self-exciting homopolar disc dynamo due to Moffatt with an additional control variable. Synchronization of two such coupled disc dynamo models is investigated by active control and sliding mode control methods. Numerical integrations show that sliding mode control provides a better synchronization in time but causes chattering. The solution is obtained by switching to active control when the synchronization errors become very small. In addition, the electronic circuit of the four-dimensional hyperchaotic system has been realized in ORCAD-PSpice and on the oscilloscope by amplitude values, verifying the results from the numerical experiments.

\section{Introduction}

Hyperchaos is a feature of a chaotic system having more than one positive Lyapunov exponent [1]. Because of potential theoretical and practical applications in technology, such as secure communications, lasers, nonlinear circuits, neural networks, generation, control, and synchronization, hyperchaos has featured as an important research area in nonlinear science [2-5]. The theory about hidden hyperchaos with either only stable or no equilibrium states is still in its infancy and has only recently been understood by mathematicians [6-9].

In 1979, Moffatt identified inconsistencies in the modeling of a simple self-exciting homopolar disc dynamo because of the neglect of induced azimuthal eddy currents, which can be resolved by introducing a segmented disc dynamo [10].
Here we investigate hidden hyperchaos, synchronization, and electronic circuit realization for a higher-dimensional version of the self-exciting homopolar disc dynamo, which was not yet completely well understood.

Since Pecora and Carroll [11] investigated synchronization in chaotic systems in 1990, such behavior has become an important research area in nonlinear science, not only for understanding the complicated phenomena in various fields but also for its potential applications especially in secure communication and image encryption. Two indistinguishable chaotic systems, starting from nonidentical initial values, would evolve in time to completely different trajectories because of the sensitive dependence of chaotic systems to their initial values. The aim of synchronizing chaos is to ensure that the states track the desired trajectory. Many effective methods exist to deal with synchronization of chaotic and 
hyperchaotic systems. These include active control [12-20], passive control [21], sliding mode control [22-31], adaptive control [32], and backstepping design [33]. Of these, active control is an important simple method used in the synchronization of nonlinear systems. It maintains asymptotic stability at zero error by eliminating the nonlinear terms and making all the eigenvalues have negative real parts. The other commonly preferred method, sliding mode control, maintains the synchronization by enforcing the error system to stay on a constructed sliding surface.

The first model with a simple electronic application was realized by Chua et al. [34]. In the following years, many electronic circuit applications such as simple RLC, RC circuits [35-37], oscillators [38, 39], power circuits [40, 41], and capacitor circuits which show chaotic features were improved upon. On the one hand, numerous electronic circuit realizations with interesting features, which mimic novel chaotic and hyperchaotic systems, have been proposed in recent years [3, 42-45].

Current interest in hidden hyperchaotic attractors motivates us to study an extension about the self-exciting homopolar disc dynamo [10] to 4D homopolar dynamo without equilibria. The existence of hidden hyperchaotic attractors in this new disc dynamo is confirmed. Synchronization of two such coupled $4 \mathrm{D}$ self-exciting homopolar disc dynamo systems is analyzed with active and sliding mode control methods. Moreover, we have designed an electronic circuit and have used an oscilloscope to view the hyperchaotic rescaled dynamo without equilibria, implemented in real time.

\section{Model and Hidden Hyperchaos of 4D Self-Exciting Homopolar Disc Dynamo System without Equilibria}

Dynamo models have been the object of much interest in order to understand both the generation of magnetic fields and their reversals in astrophysics. Moffatt [10] extended the simplest self-exciting Bullard dynamo to include radial magnetic diffusion, to produce the disc dynamo model, written nondimensionally as

$$
\begin{aligned}
& \dot{x}=r(y-x), \\
& \dot{y}=m x-(1+m) y+x z, \\
& \dot{z}=g\left[1+m x^{2}-(1+m) x y\right],
\end{aligned}
$$

where $x, y$, and $z$ are state variables and $r, m$, and $g$ are positive parameters. $x$ and $y$ represent the magnetic fluxes due to radial and azimuthal current distributions, respectively. $z$ denotes the angular velocity of the disc. $g$ is the applied torque; $r$ and $m$ are the constants which depend on the inductances and the electrical resistance of the dynamo.

By modifying the characteristics of the segmented disc dynamo (1), hidden chaotic or hyperchaotic spiral attractors have been observed numerically under special initial conditions with two symmetric stable node-foci. This leads to the interesting and striking observation of multiple attractors for

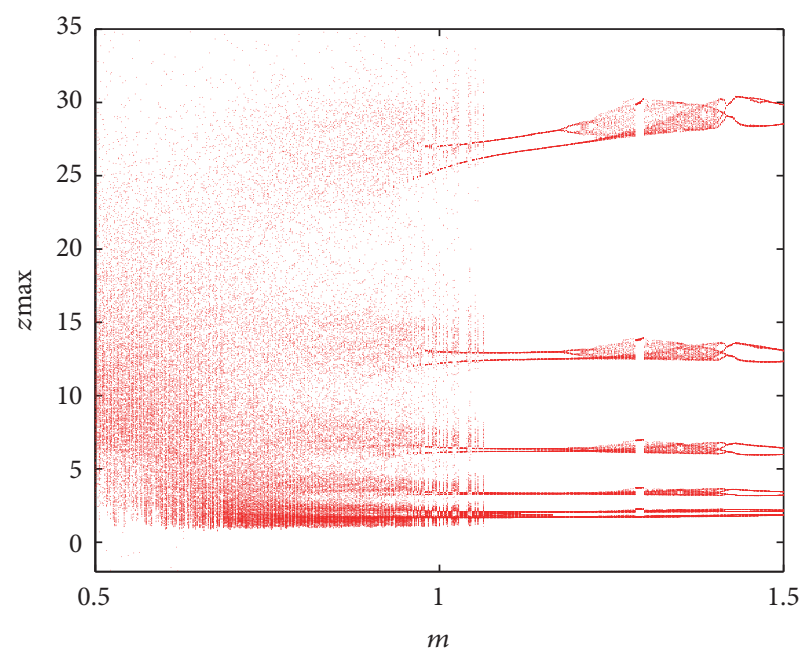

FIGURE 1: Bifurcation diagram of system (2) without equilibria versus parameter $m$ in $[0.5,1.5]$.

a broad range of parameters. As in many nonlinear dynamical systems, the occurrence of multiple attractors implies the existence of multistability in the self-exciting dynamo, with the long-term behavior being fundamentally different depending on which basin of attraction the initial conditions belong. Now, we introduce a dislocated feedback controller to system (1) as a new state $w$ and translate $z$ to $z-m$ to result in following $4 \mathrm{D}$ system:

$$
\begin{aligned}
\dot{x} & =r(y-x)+w, \\
\dot{y} & =-(1+m) y+x z, \\
\dot{z} & =g\left[1+m x^{2}-(1+m) x y\right], \\
\dot{w} & =-k y,
\end{aligned}
$$

where $k$ is a positive parameter. Although system (2) is similar to the algebraic forms of hyperchaotic Lorenz, Chen, Lü, and unified systems, they are not topologically equivalent [4652]. The proposed system (2) will be a way of understanding the generation of magnetic fields and their reversals in the Earth, the Sun, and other astrophysical bodies. Figure 1 shows a bifurcation diagram exhibiting a period-doubling route to chaos of the peak of $z$ ( $z \max )$ of system (2) versus the parameters $m \in[0.5,1], r=8, g=35$, and $k=3$. There are some periodic windows in the chaotic region. Plots of the Lyapunov exponents about $m \in[0.5,1.5]$ are shown in Figure 2. Figure 3 indicates that system (2) is indeed hidden hyperchaotic for initial states $(1.13,0.5,0.8$, and 1.5) and parameter $m=0.5$. Its Lyapunov exponents are $0.4113,0.2233$, 0.0000 , and -10.1345$)$ and Kaplan-Yorke dimension is $D_{\mathrm{KY}}=$ 3.0626 .

To find the equilibrium states of system (2), we set $\dot{x}=$ $\dot{y}=\dot{z}=\dot{w}=0$ and solve

$$
\begin{aligned}
r(y-x)+w & =0, \\
-(1+m) y+x z & =0, \\
g\left[1+m x^{2}-(1+m) x y\right] & =0, \\
-k y & =0 .
\end{aligned}
$$




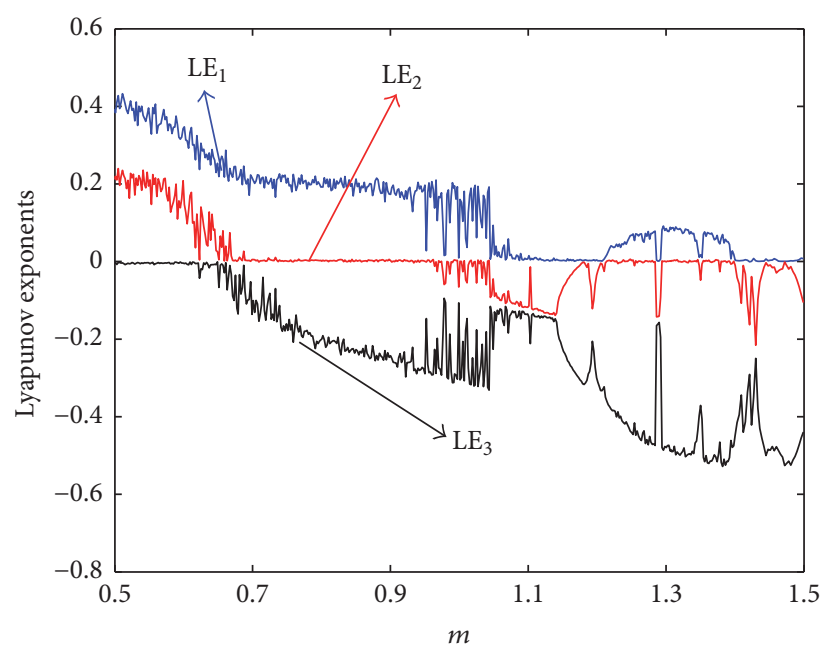

(a)

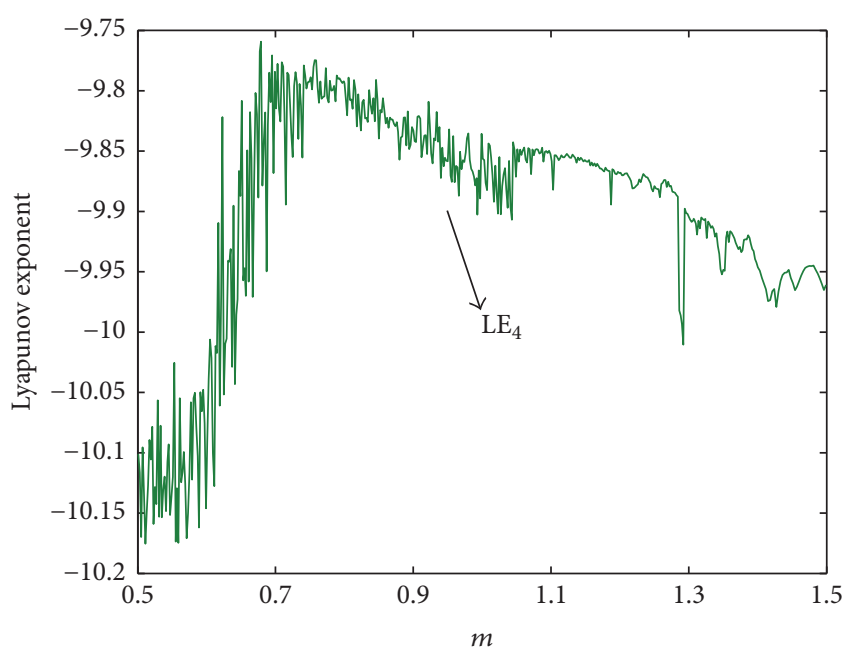

(b)

FIGURE 2: Lyapunov exponents $\mathrm{LE}_{1}, \mathrm{LE}_{2}, \mathrm{LE}_{3}$, and $\mathrm{LE}_{4}$ of system (2) without equilibria versus parameter $m$ in $[0.5,1.5]$.

There seems to be two equilibria: $E_{1}(1 / \sqrt{-m}, 0,0$, $r / \sqrt{-m})$ and $E_{2}(-1 / \sqrt{-m}, 0,0,-r / \sqrt{-m})$; however, because $m$ is positive, hyperchaotic self-exciting homopolar disc dynamo system (2) has no real equilibria.

\section{Synchronization of the 4D Self-Exciting Homopolar Disc Dynamo System}

For synchronization, two self-exciting homopolar disc dynamo hyperchaotic systems are coupled together with different initial values. The driver system, $x$, controls the response system, $y$. They are given, respectively, by

$$
\begin{aligned}
& \dot{x_{1}}=r\left(x_{2}-x_{1}\right)+x_{4}, \\
& \dot{x_{2}}=-(1+m) x_{2}+x_{1} x_{3}, \\
& \dot{x_{3}}=g\left[1+m x_{1}^{2}-(1+m) x_{1} x_{2}\right], \\
& \dot{x_{4}}=-k x_{2}, \\
& \dot{y_{1}}=r\left(y_{2}-y_{1}\right)+y_{4}, \\
& \dot{y_{2}}=-(1+m) y_{2}+y_{1} y_{3}+u_{1}, \\
& \dot{y}_{3}=g\left[1+m y_{1}^{2}-(1+m) y_{1} y_{2}\right]+u_{2}, \\
& \dot{y}_{4}=-k y_{2},
\end{aligned}
$$

where $u_{1}$ and $u_{2}$ in system (5) are the control functions to be determined. The synchronization errors are obtained by subtracting the driver and response systems. Thus, they are defined as $e_{i}=y_{i}-x_{i}($ for $i=1,2,3,4)$ and the error dynamics become as follows:

$$
\begin{aligned}
& \dot{e_{1}}=r\left(e_{2}-e_{1}\right)+e_{4}, \\
& \dot{e_{2}}=-(1+m) e_{2}+y_{1} y_{3}-x_{1} x_{3}+u_{1}, \\
& \dot{e_{3}}=g\left[m\left(y_{1}^{2}-x_{1}^{2}\right)-(1+m)\left(y_{1} y_{2}-x_{1} x_{2}\right)\right]+u_{2}, \\
& \dot{e_{4}}=-k e_{2} .
\end{aligned}
$$

Our objective is to make system (6) asymptotically stable at the zero error state.

3.1. Active Control. The nonlinear terms in system (6) can be eliminated by defining the control functions $u_{1}$ and $u_{2}$ as in the following:

$$
\begin{aligned}
& u_{1}=-y_{1} y_{3}+x_{1} x_{3}+v_{1}, \\
& u_{2}=-g\left[m\left(y_{1}^{2}-x_{1}^{2}\right)-(1+m)\left(y_{1} y_{2}-x_{1} x_{2}\right)\right]+v_{2} .
\end{aligned}
$$

Then, substituting (7) into system (6) gives

$$
\begin{aligned}
& \dot{e_{1}}=r\left(e_{2}-e_{1}\right)+e_{4}, \\
& \dot{e_{2}}=-(1+m) e_{2}+v_{1}, \\
& \dot{e_{3}}=v_{2}, \\
& \dot{e_{4}}=-k e_{2} .
\end{aligned}
$$

This implies that the equations in system (8) are linear. Provided that the proper choices of control inputs $v_{1}$ and $v_{2}$ stabilize the error system (8), then $e_{1}, e_{2}, e_{3}$, and $e_{4}$ will converge to zero as $t \rightarrow+\infty$. Then, the synchronization of two identical self-exciting homopolar disc dynamo hyperchaotic 

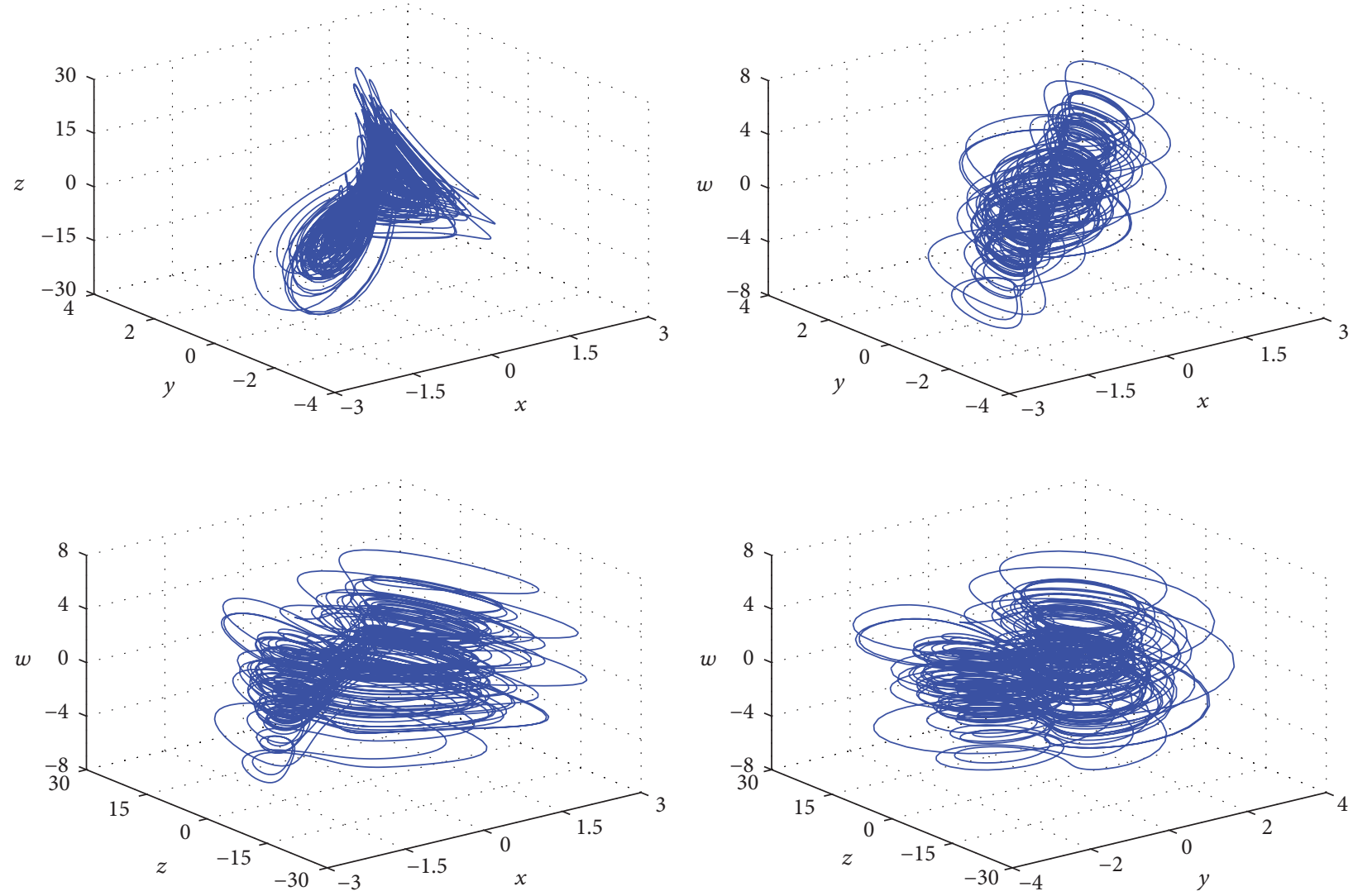

FiguRE 3: Hyperchaotic attractor of four-dimensional self-exciting homopolar disc dynamo system (2) without equilibria for $r=8, g=35$, $k=3$, and $m=0.5$.

systems will be achieved. A number of choices are possible for control functions $v_{1}$ and $v_{2}$. They are simply taken as

$$
\begin{aligned}
& v_{1}=\left(1+m-k_{1}\right) e_{2}-r e_{1}+k e_{4}, \\
& v_{2}=-k_{2} e_{3},
\end{aligned}
$$

where $k_{1}$ and $k_{2}$ are positive control gains. Substituting (9) to system (8) gives the following synchronization error dynamics:

$$
\begin{aligned}
& \dot{e_{1}}=r\left(e_{2}-e_{1}\right)+e_{4}, \\
& \dot{e_{2}}=-r e_{1}-k_{1} e_{2}+k e_{4}, \\
& \dot{e_{3}}=-k_{2} e_{3}, \\
& \dot{e_{4}}=-k e_{2} .
\end{aligned}
$$

The associated characteristic matrix of system (10) is

$$
A=\left(\begin{array}{cccc}
-r & -r & 0 & 1 \\
-r & -k_{1} & 0 & k \\
0 & 0 & -k_{2} & 0 \\
0 & -k & 0 & 0
\end{array}\right)
$$

For the particular choice of control functions in (9), the closed loop system (10) has all of its eigenspectrum in the negative half plane since all of the parameters $r, k, k_{1}$, and $k_{2}$ are positive. So, this choice leads to a stable system where the error states $e_{1}, e_{2}, e_{3}$, and $e_{4}$ tend to zero as time $t$ tends to infinity. Synchronization of two identical hyperchaotic selfexciting homopolar disc dynamos is therefore completed with the active control method.

3.2. Sliding Mode Control. It can be seen from system (6) that when $e_{2}$ and $e_{3}$ become zero, $\dot{e_{4}}$ will be zero and then $\dot{e_{1}}=$ $-r e_{1}$. Therefore, when time goes to infinite, $e_{1}$ will converge to zero, too. Appropriate sliding surfaces for $e_{2}$ and $e_{3}$ can be, respectively, designed as

$$
\begin{aligned}
& s_{1}=e_{2}-k_{3} e_{4}, \\
& s_{2}=e_{3}-k_{4} e_{4},
\end{aligned}
$$

where $k_{3}$ and $k_{4}$ are positive control gains.

The attainability condition for sliding mode is $s \dot{s}<0$. Provided that this condition is satisfied, the sliding mode control functions are

$$
\begin{aligned}
u_{1}= & (1+m) e_{2}-y_{1} y_{3}+x_{1} x_{3}-k_{3} k e_{2}-k_{5} s_{1} \\
& -q_{1} \operatorname{sign}\left(s_{1}\right), \\
u_{2}= & -g\left[m\left(y_{1}^{2}-x_{1}^{2}\right)-(1+m)\left(y_{1} y_{2}-x_{1} x_{2}\right)\right] \\
& -k_{4} k e_{2}-k_{6} s_{2}-q_{2} \operatorname{sign}\left(s_{2}\right),
\end{aligned}
$$



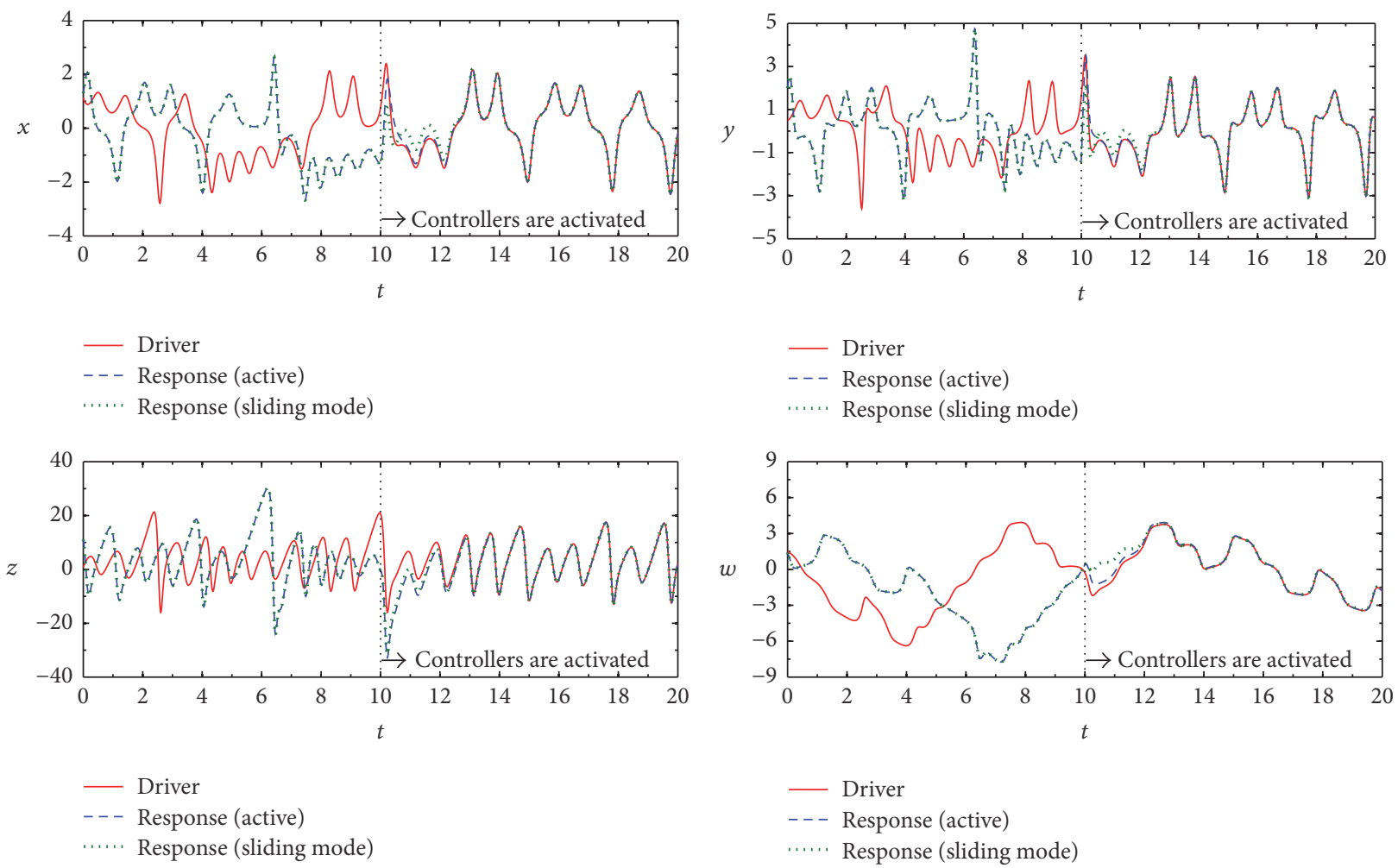

Figure 4: Time series of driver and response four-dimensional self-exciting homopolar disc dynamo hyperchaotic systems with the controllers are activated at $t=10$.

where $k_{5}$ and $k_{6}$ are positive control gains. Large values of $k_{5}$ and $k_{6}$ decrease the time $t$ taken to reach the sliding surface but lead to chattering; small values of $q_{1}$ and $q_{2}$ reduce chattering but increase the time to reach the sliding surface. Here "sign" means the signum function.

The designed control functions (13) guarantee that system (6) is held on the sliding surface $s=0$. The time derivations of sliding surfaces are

$$
\begin{aligned}
& \dot{s_{1}}=-k_{5} s_{1}-q_{1} \operatorname{sign}\left(s_{1}\right), \\
& \dot{s_{2}}=-k_{6} s_{2}-q_{2} \operatorname{sign}\left(s_{2}\right) .
\end{aligned}
$$

For the Lyapunov function

$$
V=\frac{1}{2}\left(s_{1}^{2}+s_{2}^{2}\right)
$$

the time derivative of $V$ becomes

$$
\dot{V}=-k_{5} s_{1}^{2}-q_{1} s_{1} \operatorname{sign}\left(s_{1}\right)-k_{6} s_{2}^{2}-q_{2} s_{2} \operatorname{sign}\left(s_{2}\right) \leq 0,
$$

where $k_{5,6} \geq 0$. These conditions guarantee that the constructed sliding surfaces $s_{1}$ and $s_{2}$ would asymptotically stabilize to the zero synchronization error state, and we obtain synchronization between the two identical hyperchaotic selfexciting homopolar disc dynamos via the sliding mode control method.

3.3. Numerical Simulations. We now perform some numerical experiments to show that synchronization occurs. We use an ode45 integration solver function with a variable step size. We take $r=8, m=0.5, g=35$, and $k=3$ to ensure that hyperchaotic behavior occurs. The gains of the active controllers are taken to be $k_{1}=1$ and $k_{2}=1$. The gains of the sliding mode controllers are taken to be $k_{3}=1$, $k_{4}=1, k_{5}=1, k_{6}=1, q_{1}=0.5$, and $q_{2}=0.5$. We choose the initial conditions for the driver and response systems to be $(1.13,0.5,0.8,1.5)$ and $(1.3,2,11.1,1.4)$, respectively. The controllers are activated when $t=10$, and we plot the synchronization simulations in Figure 4, while the synchronization errors are plotted in Figure 5.

Figure 4 shows that both active and sliding mode controllers achieve the synchronization of the four-dimensional self-exciting homopolar disc dynamo hyperchaotic system. Figure 5 also shows that, after the activation of controllers at $t=10$, the synchronization errors approach zero asymptotically, thereby validating the theoretical analyses. Synchronization is complete for $t \geq 16$ with active control and for $t \geq 14$ with sliding mode control. Activation of control at different times gives similar synchronization performances. The comparisons point out that the sliding mode control scheme has the advantage of a faster synchronization time. However, when the results are viewed more closely, as in Figure 6, the chattering problem of sliding mode controllers is evident. A solution is to switch to active control when the mean squared error is less than 0.00001 . The new results from switching controllers are presented in Figure 7. It has no chattering and the synchronization performance is similar to that with the sliding mode control scheme. 


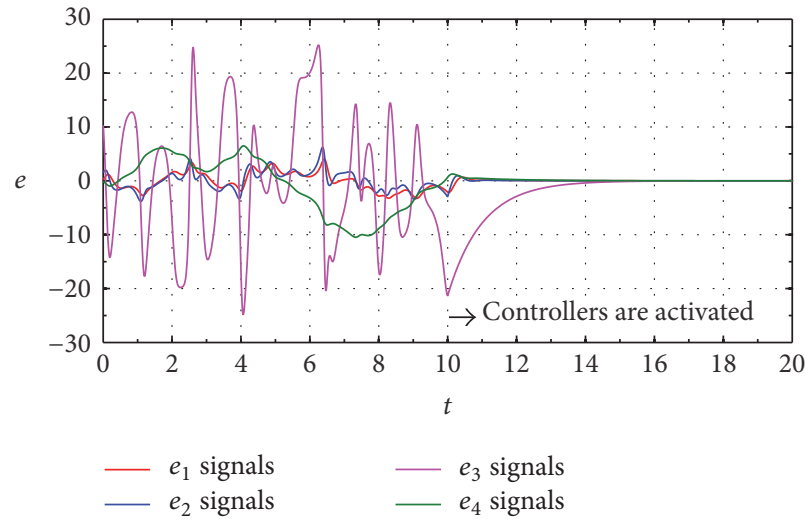

(a)

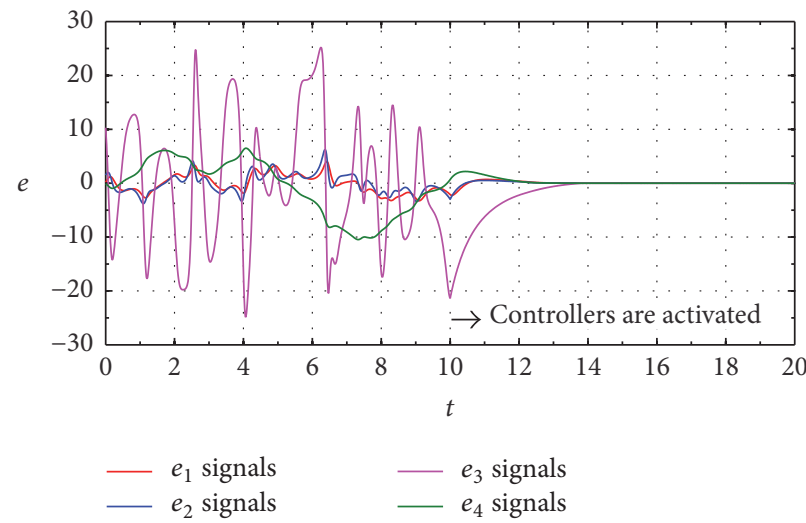

(b)

FIGURE 5: Synchronization errors between driver and response four-dimensional self-exciting homopolar disc dynamo hyperchaotic systems with the controllers are activated at $t=10$ : (a) active controllers and (b) sliding mode controllers.

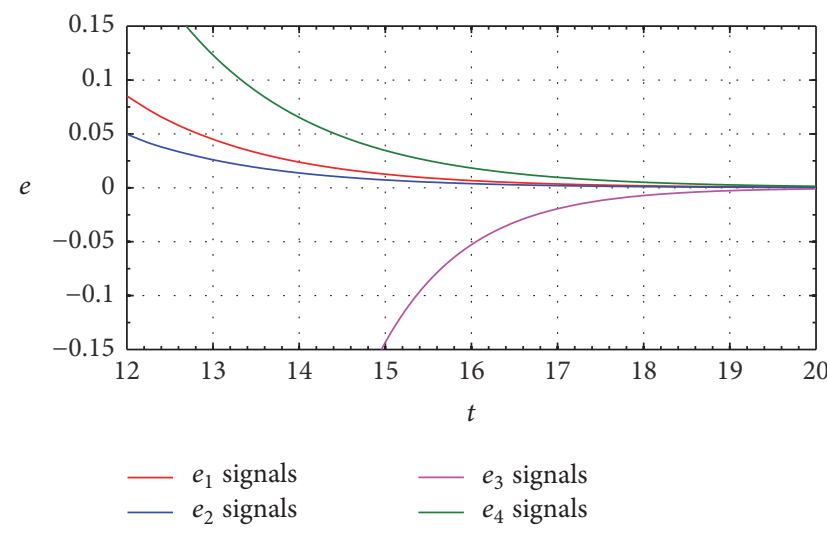

(a)

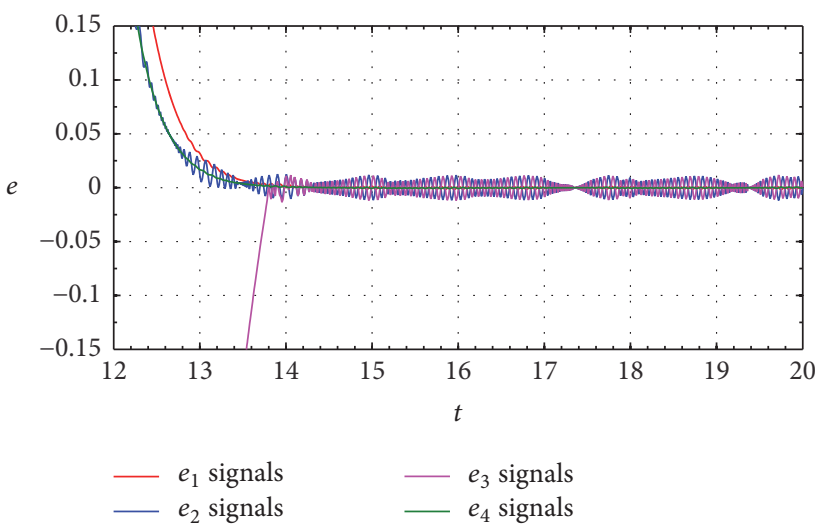

(b)

FIGURE 6: Synchronization errors between $t \geq 12$ and $t \leq 20$ : (a) active controllers and (b) sliding mode controllers.

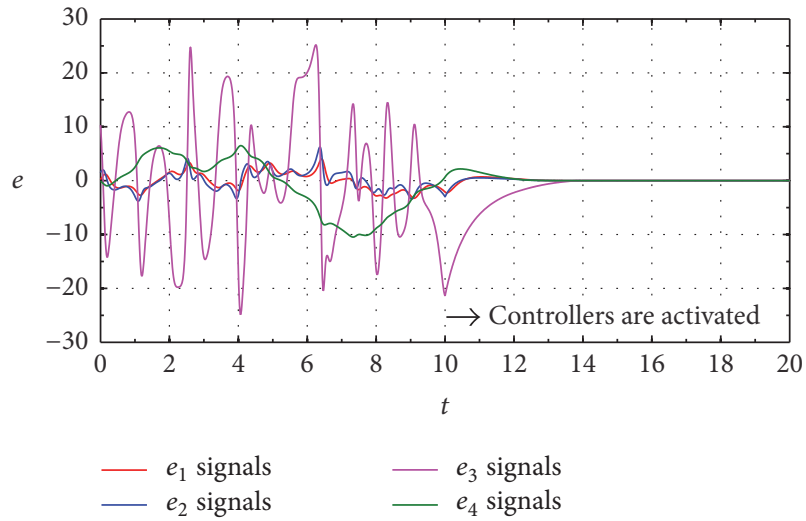

(a)

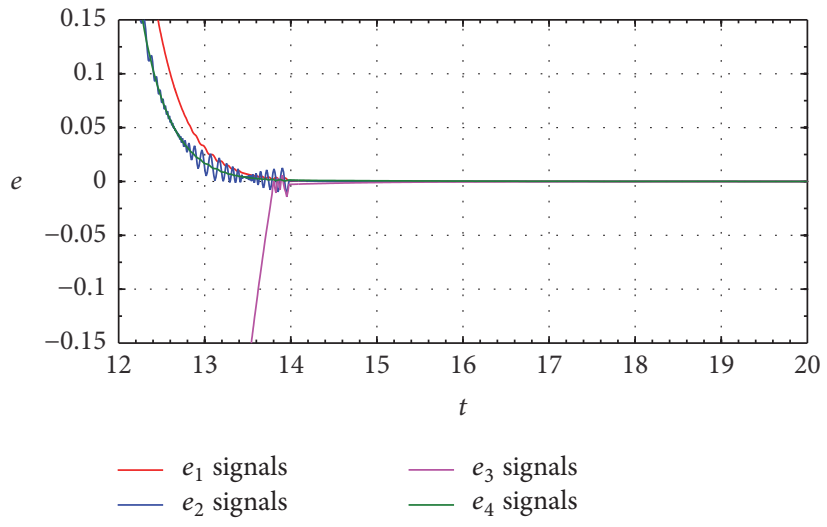

(b)

FIGURE 7: Synchronization errors with the proposed switching controllers are activated: (a) $t \leq 20$; (b) $t \geq 12, t \leq 20$. 


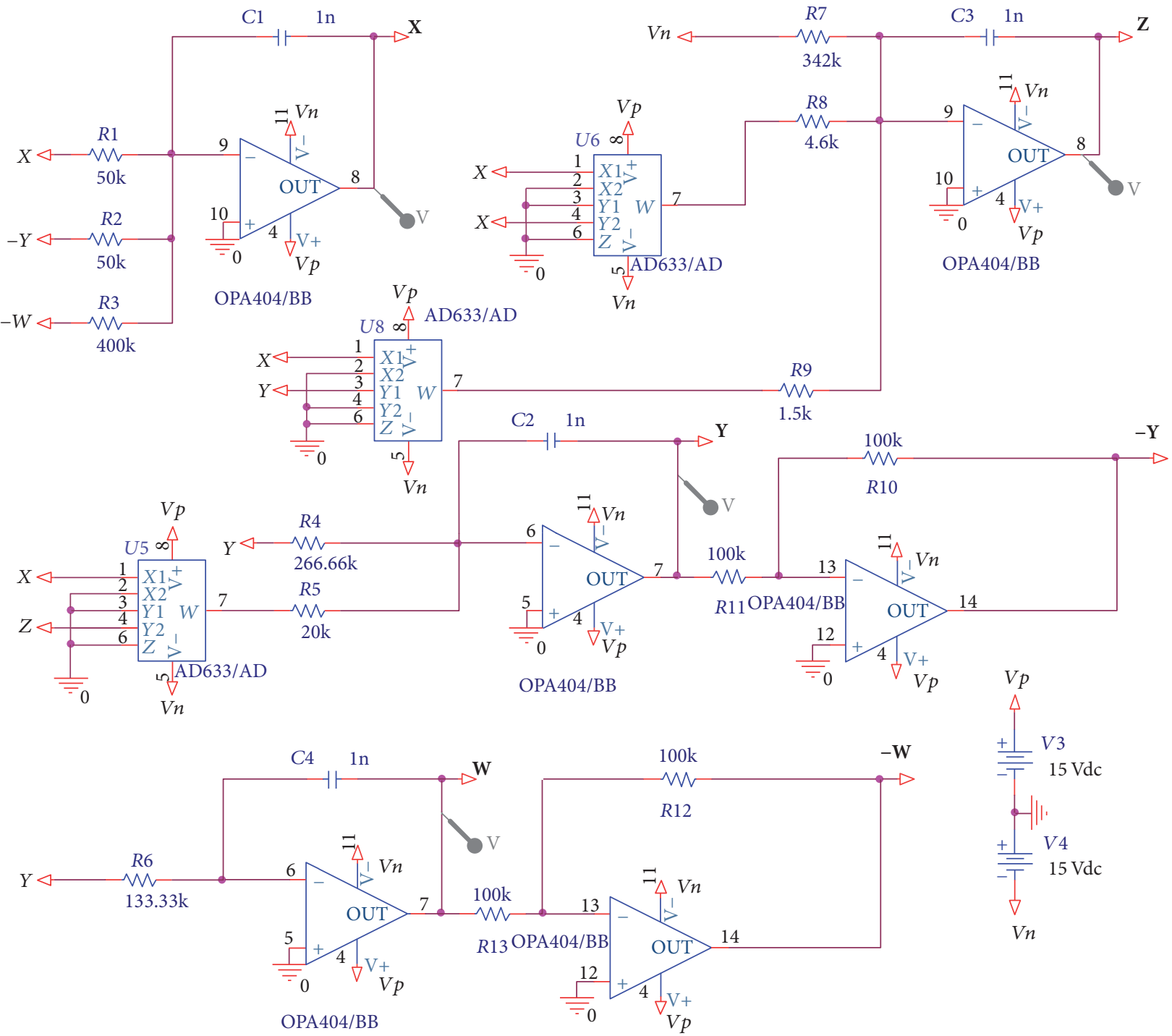

FIGURE 8: The electronic circuit schematic of the scaled hyperchaotic system (17).

\section{Electronic Circuit Implementation of the 4D Self-Exciting Homopolar Disc Dynamo System}

Because the values for $x, y$, and $w$ fall within the interval of $(-15,15)$, while $z$ falls within the interval of $(-20,25), z$ must be rescaled for observations using the oscilloscope. We let $X=x, Y=y, Z=z / 2$, and $W=w$ and consider the system:

$$
\begin{aligned}
\dot{X} & =r(Y-X)+W, \\
\dot{Y} & =-(1+m) Y+2 X Z, \\
\dot{Z} & =\frac{1}{2} g\left[1+m X^{2}-(1+m) X Y\right], \\
\dot{W} & =-k Y .
\end{aligned}
$$

We can now design an electronic circuit for the scaled hyperchaotic model (17) with parameters $r=8, m=0.5$, $g=35$, and $k=3$ and initial conditions $(1.13,0.5,0.4,1.5)$ using ORCAD-PSpice. The schematic of the electronic circuit is shown in Figure 8. We used resistors, capacitors, TL081 opamps, and AD633 multipliers with the values $R 1=R 2=$ $50 \mathrm{Kohm}, R 3=400 \mathrm{Kohm}, R 4=266.66 \mathrm{Kohm}, R 5=20$ Kohm, $R 6=133.33 \mathrm{Kohm}, R 7=342 \mathrm{Kohm}, R 8=4.6 \mathrm{Kohm}$, $R 9=1.5 \mathrm{Kohm}, R 10=R 11=R 12=R 13=100 \mathrm{Kohm}$, $C 1=C 2=C 3=C 4=1 \mathrm{nF}, V n=-15 \mathrm{~V}$, and $V p=15 \mathrm{~V}$. Since the AD633 multiplier IC is limited to between $-10 \mathrm{~V}$ and $+10 \mathrm{~V}$, the output voltage must be divided by $10 \mathrm{~V}$. Realtime application of system (17) was realized with electronic components and shown on the electronic card in Figure 9.

Outputs from the ORCAD-PSpice simulation and oscilloscope phase portraits for the scaled hyperchaotic system (17) with parameters $r=8, m=0.5, g=35$, and $k=3$ are given in Figures 10 and 11, respectively. The outputs verify those of the hyperchaotic system, which was modeled using MATLAB. 

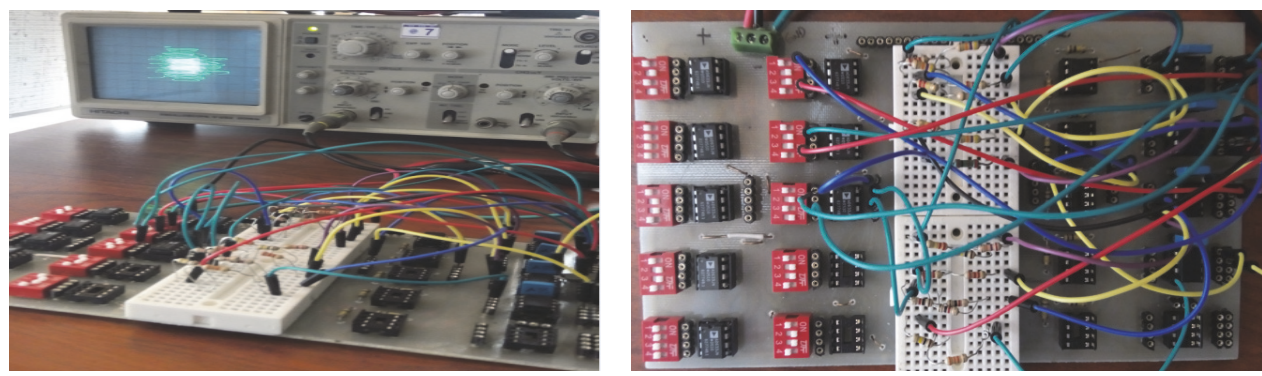

FIgURE 9: The experimental circuit of the hyperchaotic circuit.
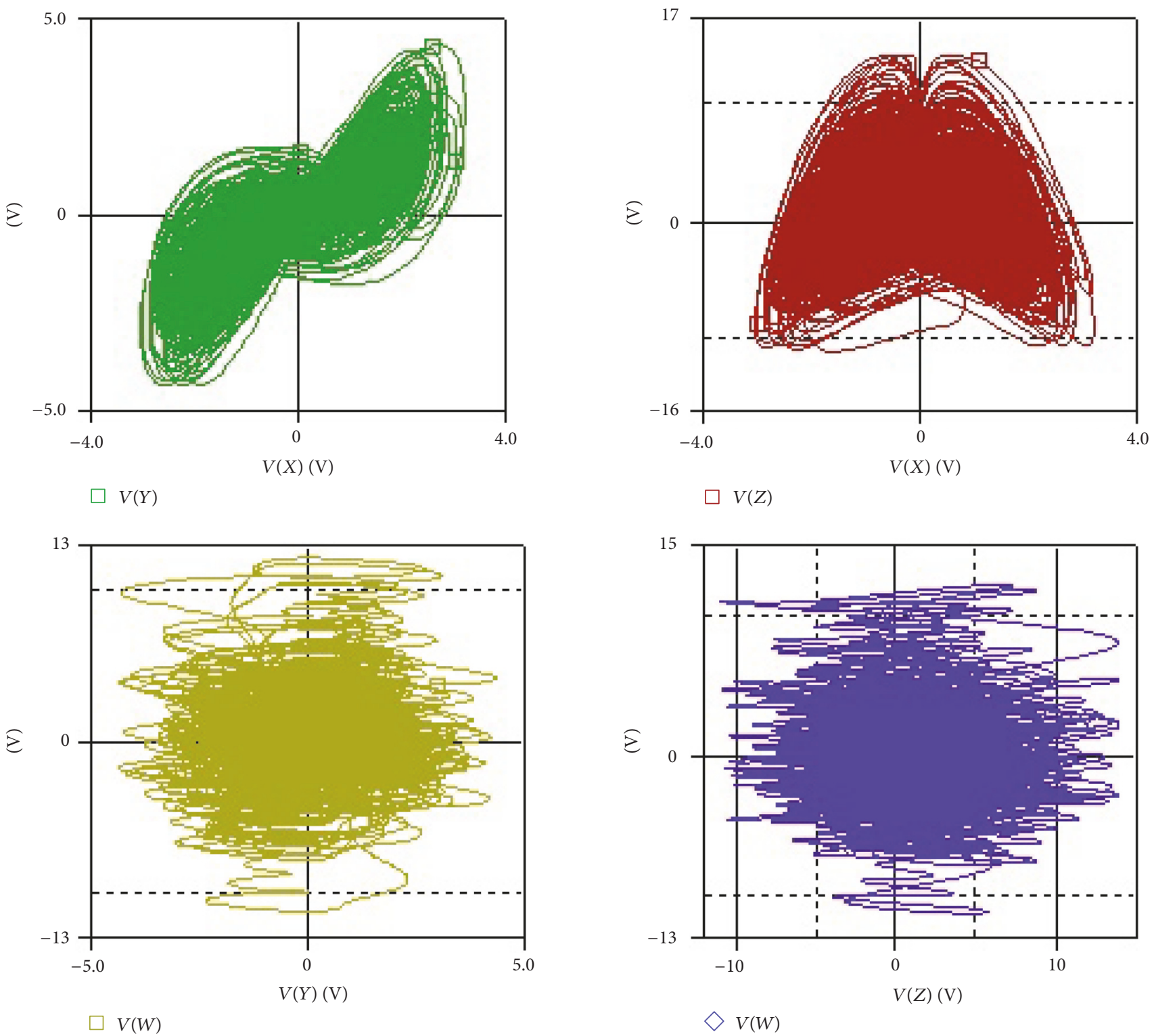

FIGURE 10: The phase portraits of scaled hyperchaotic system (17) in ORCAD-PSpice.

\section{Conclusion}

In this paper, we propose a novel four-dimensional selfexciting homopolar disc dynamo without equilibria, but exhibiting hidden hyperchaos. Furthermore, active control and sliding mode control methods are applied to synchronize two coupled four-dimensional dynamo systems. The feasibility of active controllers is ensured by requiring that the spectrum of eigenvalues of the synchronized error system falls in the left half plane. A Lyapunov function is proposed to 

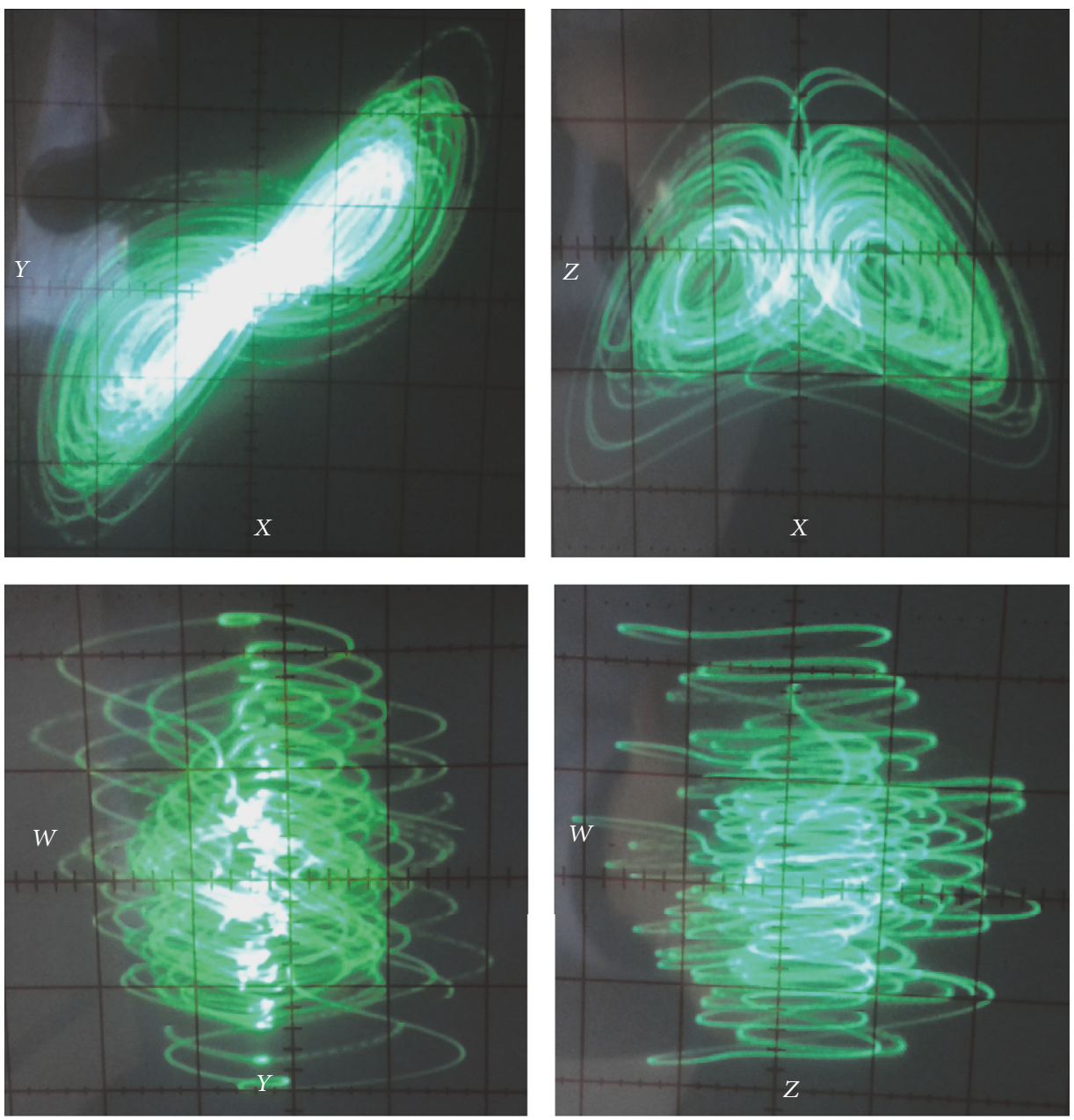

FIGURE 11: The phase portraits of scaled hyperchaotic system (17) with $r=8, m=0.5, g=35$, and $k=3$ on the oscilloscope.

guarantee the asymptotic stability of sliding surfaces and convergence to zero synchronization error. Numerical integrations are presented to compare the performances of the two controllers. Sliding mode control scheme gives better results but has chattering. Solution is provided by a switch to active controllers when chattering starts. An electronic circuit for the rescaled system is implemented via the ORCADPSpice program. Numerical simulations validated the theoretical analyses. This physical example will form the basis of more systematic studies of hyperchaos without equilibria in a future study.

\section{Conflicts of Interest}

The authors declare that they have no conflicts of interest.

\section{Acknowledgments}

This work was supported by the National Natural Science Foundation of China (Grant no. 11561069), the Guangxi Natural Science Foundation of China (Grant no. 2016GXNSFBA380170), the Scientific Research Foundation of the Higher Education Institutions of Guangxi Province of China (Grant no. KY2016YB364), the Open Foundation for Guangxi Colleges and Universities Key Lab of Complex System Optimization and Big Data Processing (nos. 2016CSOBDP0003 and 2016CSOBDP0202), and Sakarya University Scientific Research Projects Unit (no. 201609-00008).

\section{References}

[1] O. E. Rössler, "An equation for hyperchaos," Physics Letters A, vol. 71, no. 2-3, pp. 155-157, 1979.

[2] P. Arena, S. Baglio, L. Fortuna, and G. Manganaro, "Hyperchaos from cellular neural networks," Electronics Letters, vol. 31, no. 4, pp. 250-251, 1995.

[3] Q. Li, S. Tang, H. Zeng, and T. Zhou, "On hyperchaos in a small memristive neural network," Nonlinear Dynamics, vol. 78, no. 2, pp. 1087-1099, 2014.

[4] L. Liu, C. Liu, and Y. Zhang, "Theoretical analysis and circuit implementation of a novel complicated hyperchaotic system," Nonlinear Dynamics, vol. 66, no. 4, pp. 707-715, 2011. 
[5] C. B. Li, I. Pehlivan, J. C. Sprott, and A. Akgul, "A novel fourwing strange attractor born in bistability," IEICE Electronics Express, vol. 12, no. 4, pp. 1-12, 2015.

[6] M. A. Kiseleva, N. V. Kuznetsov, and G. A. Leonov, "Hidden attractors in electromechanical systems with and without equilibria," IFAC-PapersOnLine, vol. 49, no. 14, pp. 51-55, 2016.

[7] G. A. Leonov and N. V. Kuznetsov, "Hidden attractors in dynamical systems: From hidden oscillations in Hilbert-Kolmogorov, Aizerman and Kalman problems to hidden chaotic attractor in Chua circuits," International Journal of Bifurcation and Chaos, vol. 23, no. 1, Article ID 1330002, 2013.

[8] Z. Wei, P. Yu, W. Zhang, and M. Yao, "Study of hidden attractors, multiple limit cycles from Hopf bifurcation and boundedness of motion in the generalized hyperchaotic Rabinovich system," Nonlinear Dynamics, vol. 82, no. 1-2, pp. 131-141, 2015.

[9] Z. C. Wei and W. Zhang, "Hidden hyperchaotic attractors in a modified Lorenz-Stenflo system with only one stable equilibrium," International Journal of Bifurcation and Chaos, vol. 24, no. 10, Article ID 1450127, 2014.

[10] H. K. Moffatt, "A self-consistent treatment of simple dynamo systems," Geophysical \& Astrophysical Fluid Dynamics, vol. 14, no. 1, pp. 147-166, 1979.

[11] L. M. Pecora and T. L. Carroll, "Synchronization in chaotic systems," Physical Review Letters, vol. 64, no. 8, pp. 821-824, 1990.

[12] H. N. Agiza and M. T. Yassen, "Synchronization of Rossler and Chen chaotic dynamical systems using active control," Physics Letters A, vol. 278, no. 4, pp. 191-197, 2001.

[13] E.-W. Bai and K. E. Lonngren, "Synchronization of two Lorenz systems using active control," Chaos, Solitons and Fractals, vol. 8, no. 1, pp. 51-58, 1997.

[14] M. M. El-Dessoky, "Synchronization and anti-synchronization of a hyperchaotic Chen system," Chaos, Solitons and Fractals, vol. 39, no. 4, pp. 1790-1797, 2009.

[15] A. Goksu, U. E. Kocamaz, and Y. Uyaroglu, "Synchronization and control of chaos in supply chain management," Computers and Industrial Engineering, vol. 86, pp. 107-115, 2015.

[16] G.-H. Li, "An active control synchronization for two modified Chua circuits," Chinese Physics, vol. 14, no. 3, pp. 472-475, 2005.

[17] A. Uçar, K. E. Lonngren, and E.-W. Bai, "Synchronization of the unified chaotic systems via active control," Chaos, Solitons and Fractals, vol. 27, no. 5, pp. 1292-1297, 2006.

[18] U. E. Vincent, "Synchronization of Rikitake chaotic attractor using active control," Physics Letters A, vol. 343, no. 1-3, pp. 133$138,2005$.

[19] Y. Q. Wei and N. Jiang, "Synchronization of hyperchaotic Rossler system and hyperchaotic Lorenz system with different structure," in Int. Conf. on Web Information Systems and Mining, vol. 6988, pp. 147-153, Taiyuan, China, 2011.

[20] X. B. Zhou, Y. Wu, Y. Li, and H. Q. Xue, "Synchronization of Hyperchaotic Systems via Active Control," in Proceedings of 5th International Conference on Communications, Circuits and Systems, pp. 1094-1098, Kokura, July 2007.

[21] X. Zhou, B. Kong, and H. Ding, "Synchronization and antisynchronization of a new hyperchaotic Lü system with uncertain parameters via the passive control technique," Physica Scripta, vol. 85, no. 6, Article ID 065004, 2012.

[22] C.-K. Chen, J.-J. Yan, and T.-L. Liao, "Sliding mode control for synchronization of Rössler systems with time delays and its application to secure communication," Physica Scripta, vol. 76, no. 5, pp. 436-441, 2007.
[23] Y.-Y. Hou, B.-Y. Liau, and H.-C. Chen, "Synchronization of unified chaotic systems using sliding mode controller," Mathematical Problems in Engineering, vol. 2012, Article ID 632712, 2012.

[24] U. E. Kocamaz, A. Göksu, H. Taşkın, and Y. Uyaroğlu, "Synchronization of chaos in nonlinear finance system by means of sliding mode and passive control methods: a comparative study, 'Information Technology and Control, vol. 44, no. 2, pp. 172-181, 2015.

[25] D. I. R. Almeida, J. Alvarez, and J. G. Barajas, "Robust synchronization of Sprott circuits using sliding mode control," Chaos, Solitons and Fractals, vol. 30, no. 1, pp. 11-18, 2006.

[26] Y. Toopchi and J. Wang, "Chaos control and synchronization of a hyperchaotic Zhou system by integral sliding mode control," Entropy, vol. 16, no. 12, pp. 6539-6552, 2014.

[27] S. Vaidyanathan, "Sliding mode controller design for the global chaos synchronization of rucklidge chaotic systems," International Journal on Bioinformatics \& Biosciences, vol. 2, no. 1, pp. 23-31, 2012.

[28] S. Vaidyanathan, "Sliding mode controller design for the global chaos synchronization of hyperchaotic Lü systems," International Journal on Computational Science \& Applications, vol. 2, no. 3, pp. 21-31, 2012.

[29] S. Vaidyanathan and S. Sampath, "Global chaos synchronization of hyperchaotic Lorenz systems by sliding mode control," in Proceedings of 1st Int. Conf. on Digital Image Processing and Pattern Recognition, vol. 205, pp. 156-164, Tirunelveli, India, 2011.

[30] S. Vaidyanathan and S. Sivaperumal, "Global chaos synchronization of the hyperchaotic Qi systems by sliding mode control," International Journal on Computer Science and Engineering, vol. 3, no. 6, pp. 2430-2437, 2011.

[31] J.-J. Yan, J.-S. Lin, and T.-L. Liao, "Synchronization of a modified Chua's circuit system via adaptive sliding mode control," Chaos, Solitons and Fractals, vol. 36, no. 1, pp. 45-52, 2008.

[32] S. Vaidyanathan, "Adaptive control and synchronization of hyperchaotic Newton-Leipnik system," International Journal of Advanced Information Technology, vol. 1, no. 3, pp. 22-33, 2011.

[33] Y. A. Hu and M. M. Sun, "Synchronization of a class of hyperchaotic systems via backstepping and its application to secure communication," in Proceedings of 5th Int. Conf. on Instrumentation \& Measurement, Computer, Communication, and Control, pp. 1055-1060, Computer, Communication, and Control, Qinhuangdao, China, 2015.

[34] L. O. Chua, C. W. Wu, A. Huang, and G.-Q. Zhong, "Universal circuit for studying and generating chaos-part I: routes to chaos," IEEE Transactions on Circuits and Systems I: Fundamental Theory and Applications, vol. 40, no. 10, pp. 732-744, 1993.

[35] S. Nakagawa and T. Saito, "An RC OTA hysteresis chaos generator," IEEE Transactions on Circuits and Systems I: Fundamental Theory and Applications, vol. 43, no. 12, pp. 1019-1021, 1996.

[36] M. J. Ogorzalek, "Order and chaos in a third-order RC ladder network with nonlinear feedback," IEEE Transactions on Circuits and Systems, vol. 36, no. 9, pp. 1221-1230, 1989.

[37] A. Tamaševičius, A. Namajunas, and A. Čenys, "Simple 4D chaotic oscillator," Electronics Letters, vol. 32, no. 11, pp. 957-958, 1996.

[38] H. Kawakami, "Bifurcation of periodic responses in forced dynamic nonlinear circuits: computation of bifurcation values of the system parameters," IEEE Transactions on Circuits and Systems, vol. 31, no. 3, pp. 248-260, 1984. 
[39] T. Saito, "Chaotic phenomena in a coupled oscillator," in Proceedings of European Conf. on Circuit Theory and Design., pp. 275-280, 1987.

[40] D. C. Hamill and D. J. Jeffries, "Subharmonics and chaos in a controlled switched-mode power converter," IEEE Transactions on Circuits and Systems, vol. 35, no. 8, pp. 1059-1061, 1988.

[41] G. Poddar, K. Chakrabarty, and S. Banerjee, "Control of chaos in the boost converter," Electronics Letters, vol. 31, no. 11, pp. 841$842,1995$.

[42] A. Akgul, I. Moroz, I. Pehlivan, and S. Vaidyanathan, "A new four-scroll chaotic attractor and its engineering applications," Optik, vol. 127, no. 13, pp. 5491-5499, 2016.

[43] A. Akgul and I. Pehlivan, "A new three-dimensional chaotic system without equilibrium points, its dynamical analyses and electronic circuit application," Technical Gazette, vol. 23, no. 1, pp. 209-214, 2016.

[44] A. Akgul, S. Hussain, and I. Pehlivan, "A new three-dimensional chaotic system, its dynamical analysis and electronic circuit applications," Optik, vol. 127, no. 18, pp. 7062-7071, 2016.

[45] S. Kaçar, "Analog circuit and microcontroller based RNG application of a new easy realizable $4 \mathrm{D}$ chaotic system," Optik, vol. 127, no. 20, pp. 9551-9561, 2016.

[46] Q. Jia, "Hyperchaos generated from the Lorenz chaotic system and its control," Physics Letters A, vol. 366, no. 3, pp. 217-222, 2007.

[47] G.-Q. Si, H. Cao, and Y.-B. Zhang, "A new four-dimensional hyperchaotic Lorenz system and its adaptive control," Chinese Physics B, vol. 20, no. 1, Article ID 010509, 2011.

[48] T. Gao, Z. Chen, Z. Yuan, and G. Chen, "A hyperchaos generated from Chen's system," International Journal of Modern Physics C, vol. 17, no. 4, pp. 471-478, 2006.

[49] L.-X. Jia, H. Dai, and M. Hui, "A new four-dimensional hyperchaotic Chen system and its generalized synchronization," Chinese Physics B, vol. 19, no. 10, Article ID 100501, 2010.

[50] A. Chen, J. Lu, J. Lü, and S. Yu, "Generating hyperchaotic Lü attractor via state feedback control," Physica A: Statistical Mechanics and Its Applications, vol. 364, pp. 103-110, 2006.

[51] S. Q. Pang and Y. J. Liu, "A new hyperchaotic system from the Lü system and its control," Journal of Computational and Applied Mathematics, vol. 235, no. 8, pp. 2775-2789, 2011.

[52] X. Y. Wang and G. B. Zhao, "Hyperchaos generated from the unified chaotic system and its control," International Journal of Modern Physics B, vol. 24, no. 23, pp. 4619-4637, 2010. 


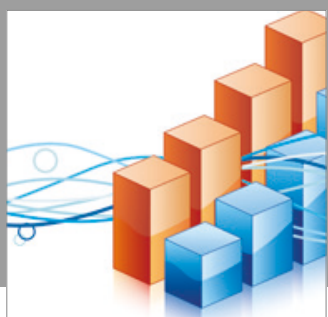

Advances in

Operations Research

vatersals

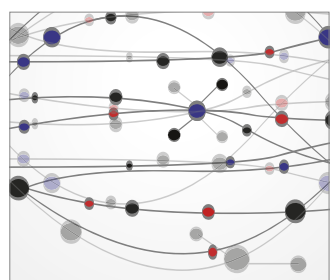

\section{The Scientific} World Journal
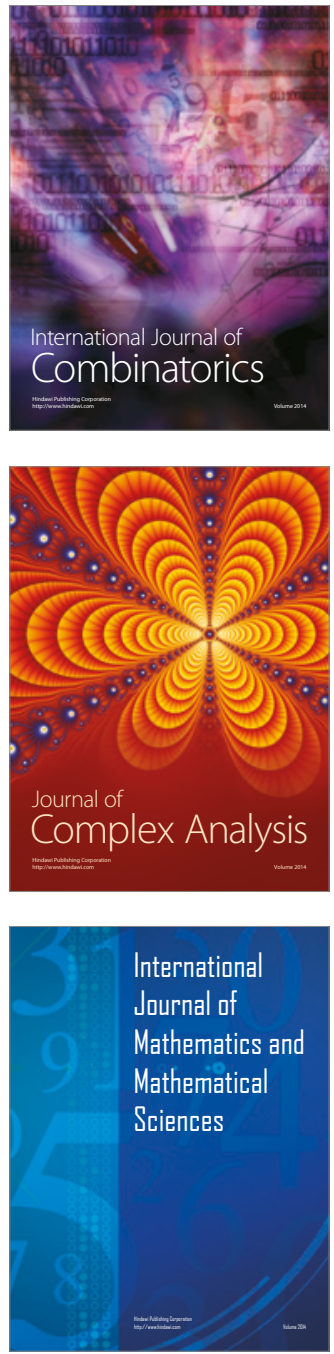
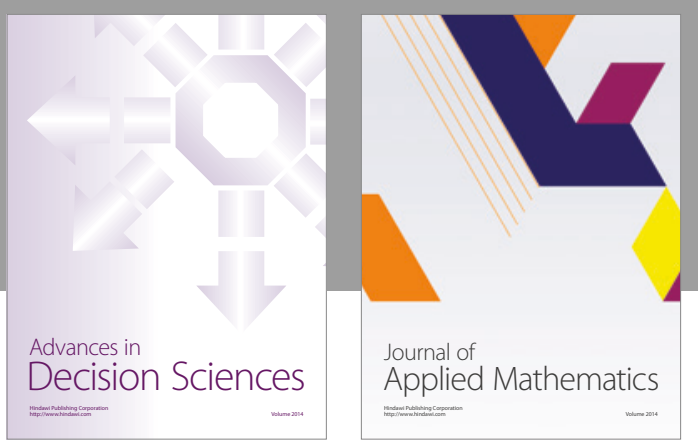

Algebra

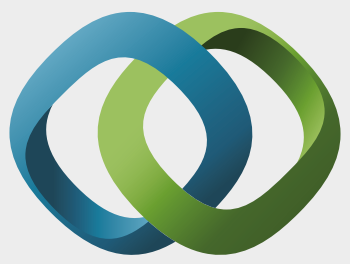

\section{Hindawi}

Submit your manuscripts at

https://www.hindawi.com
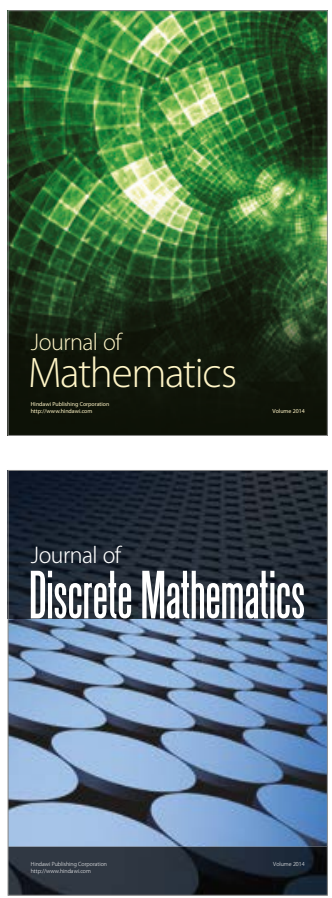

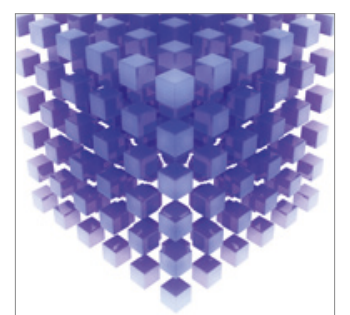

Mathematical Problems in Engineering
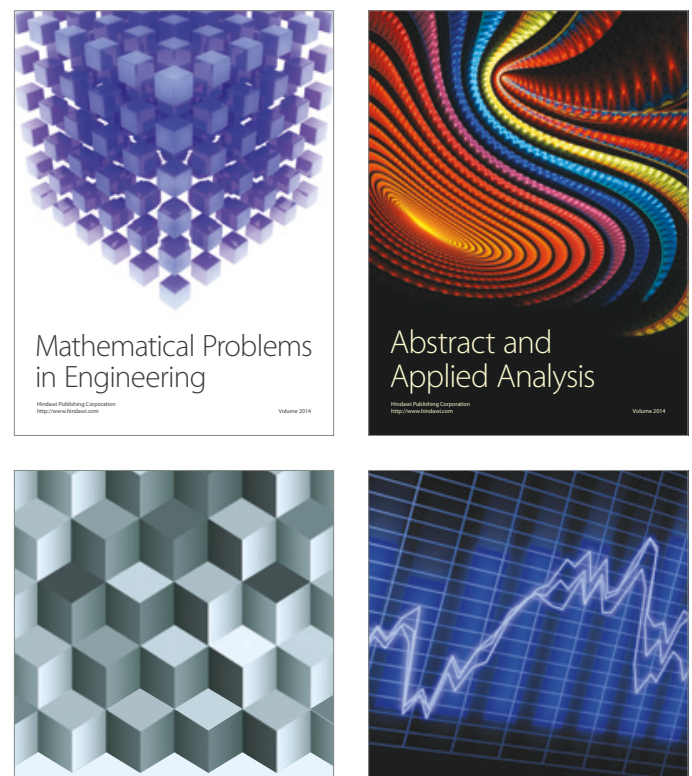

Journal of

Function Spaces

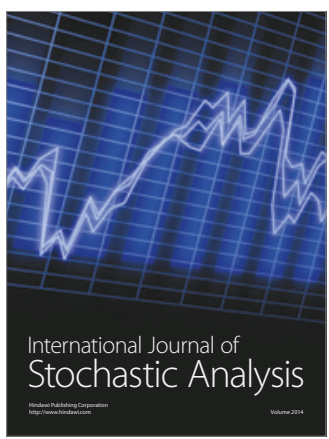

Probability and Statistics
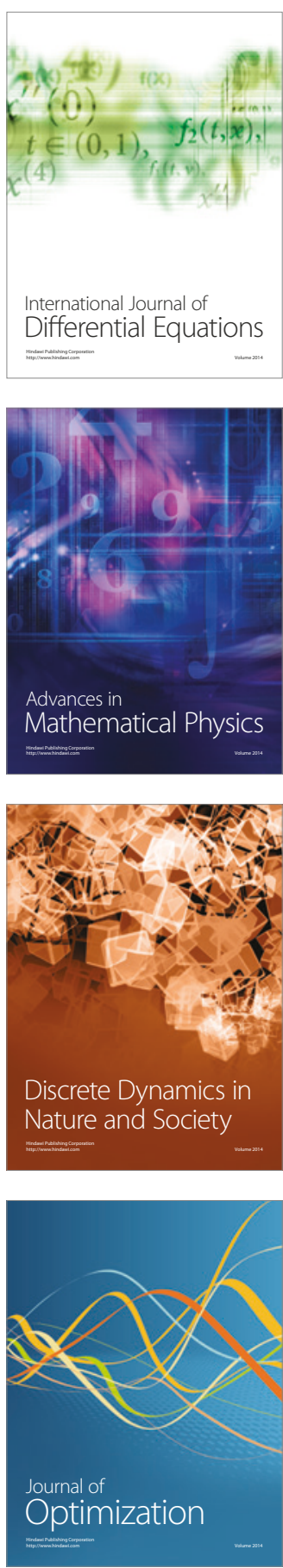